NISTIR 8127

\title{
End-to-End Quality Information Framework (QIF) Technology Survey
}

John Michaloski

Tom Hedberg

Hui Huang

Thomas Kramer

This publication is available free of charge from:

http://dx.doi.org/10.6028/NIST.IR.8127

April 2016

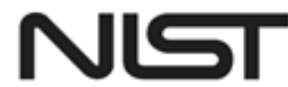

National Institute of Standards and Technology 
NISTIR 8127

\section{End-to-End Quality Information Framework (QIF) Technology Survey}

John Michaloski

Tom Hedberg

Hui Huang

Thomas Kramer

Engineering Laboratory (EL)

This publication is available free of charge from:

http://dx.doi.org/10.6028/NIST.IR.8127

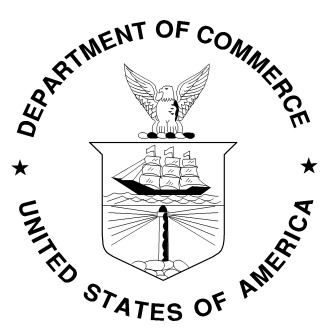

U.S. Department of Commerce

Penny Pritzker, Secretary

National Institute of Standards and Technology Willie May, Under Secretary of Commerce for Standards and Technology and Director 


\section{Table of Contents}

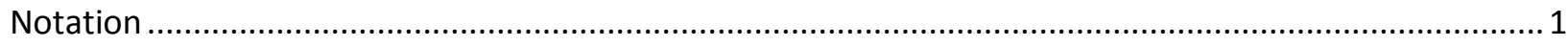

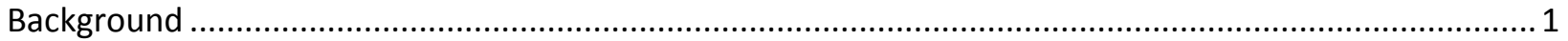

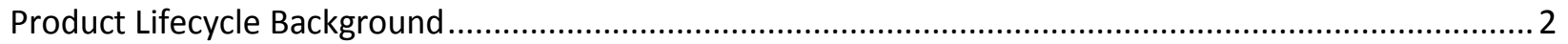

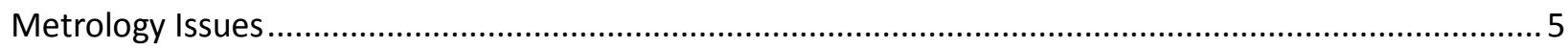

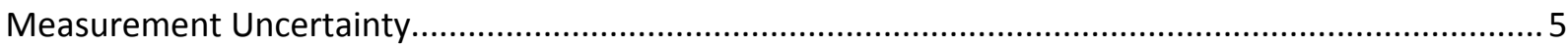

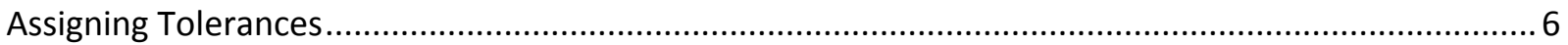

Lossless Data Exchange and Preventing Digital Tolerance Stack Up .................................................. 9

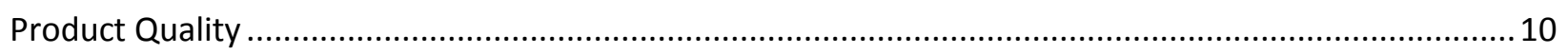

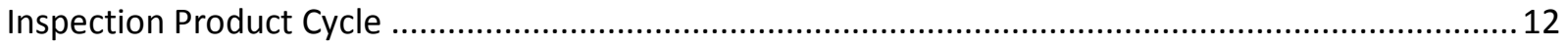

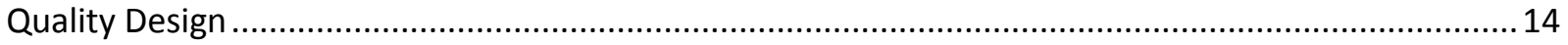

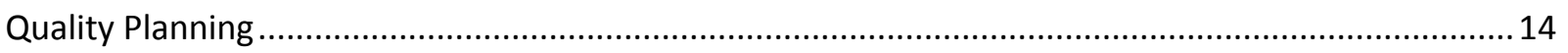

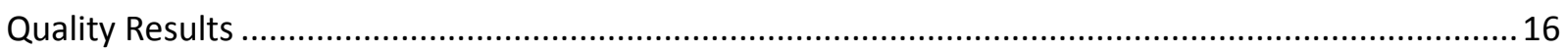

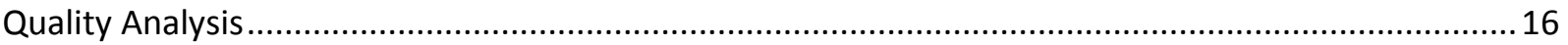

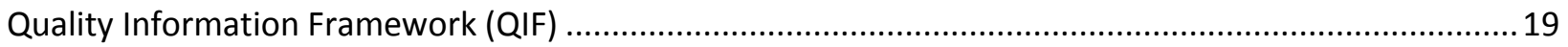

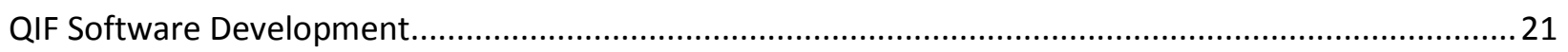

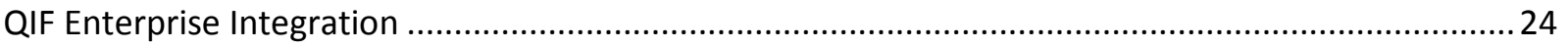

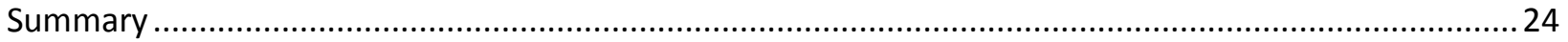

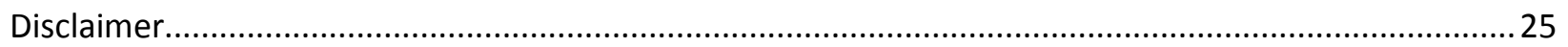

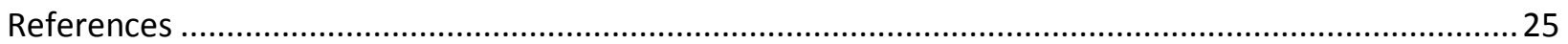




\section{Table of Figures}

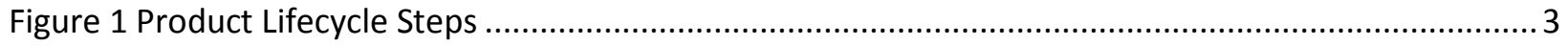

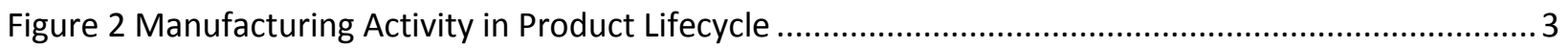

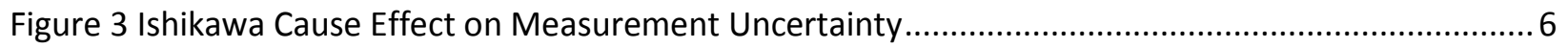

Figure 4 Tolerance Analysis ( [18] Used with Permission) .................................................................. 7

Figure 5 Activities and relations within CLTE [19] (Used with Permission) . .......................................... 8

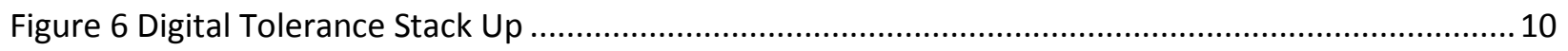

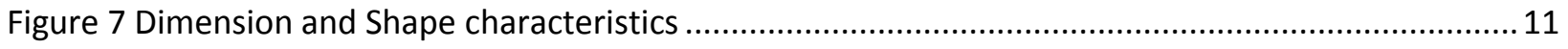

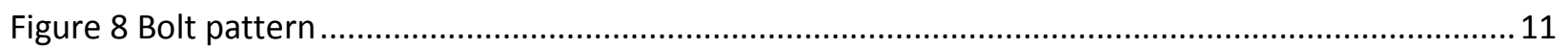

Figure 9a Example Block with Hole GD\&T Symbols ......................................................................... 12

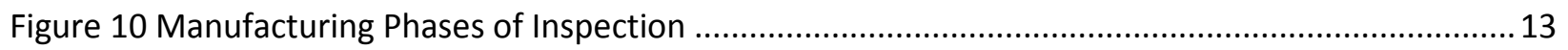

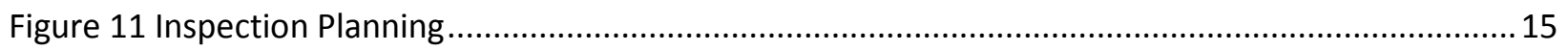

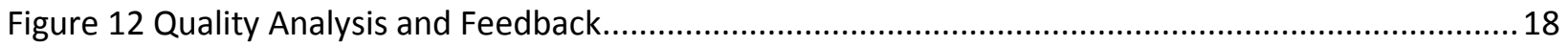

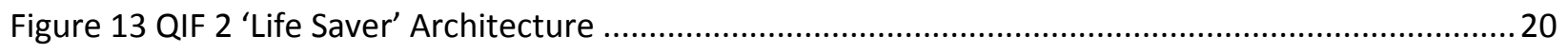

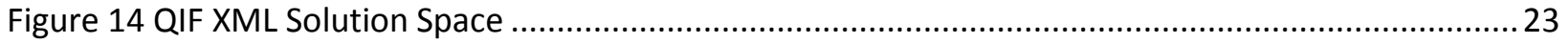




\section{Notation}

$\begin{array}{ll}\text { ANSI } & \text { American National Standards Institute } \\ \text { CAD } & \text { Computer-Aided Design } \\ \text { CAM } & \text { Computer-Aided Manufacturing } \\ \text { CAPP } & \text { Computer-Aided Process Planning } \\ \text { CAQ } & \text { Computer-Aided Quality } \\ \text { CIM } & \text { Computer Integrated Manufacturing } \\ \text { DOM } & \text { Document Object Model } \\ \text { FAIR } & \text { First Article Inspection Report } \\ \text { FEA } & \text { Finite Element Analysis } \\ \text { GD\&T } & \text { Geometric Dimensioning and Tolerancing } \\ \text { IMTS } & \text { International Manufacturing Technology Show } \\ \text { MBD } & \text { Model-Based Design } \\ \text { NIST } & \text { National Institute of Standards and Technology } \\ \text { PDF } & \text { Portable Document Format } \\ \text { PLM } & \text { Product Lifecycle Management } \\ \text { PMI } & \text { Product and Manufacturing Information } \\ \text { STEP } & \text { Standard for the Exchange of Product model data } \\ \text { QIF } & \text { Quality Information Framework } \\ \text { SME } & \text { Society of Manufacturing Engineers } \\ \text { XML } & \text { Extensible Markup Language } \\ \text { XSD } & \text { XML Schema }\end{array}$

\section{Background}

Quality of a product may be defined as "its ability to fulfill the customer's needs and expectations" [1]. Quality is defined in terms of performance requirements, which vary from product to product. For discrete parts, the primary performance requirements, commonly referred to as characteristics, are with respect to dimension (e.g., length, diameter, thickness, or area), geometry (e.g., flatness, cylindricity, etc.), and appearance (e.g., surface finish, color, or texture). To ensure overall quality, delivered parts must meet the required quality characteristics. Thus, part quality is measured by its conformance to the performance requirements.

Intuition, "rules of thumb", and educated guesses are ineffective ways to guarantee part quality. Quality goods can only be manufactured using hard knowledge derived from superior part design, part fabrication, and ongoing and continuous collection and evaluation of production data. Of interest in our work is the use of quality standards, and especially feedback through quality standards, to improve part design and fabrication as a consequence of the quality reporting of products.

Manufacturing systems are by their nature imperfect. Process variability can hinder manufacturers in their effort to maintain acceptable part quality. However, inadequate control of process variability can lead to scrap, rework, and repair. Maintaining close control of production quality requires measurement and adjustment to design and manufacturing process errors. Inspection is used to provide insight and visibility to potential production problems so that they can be rectified in a timely manner. Inspection is an integral part of manufacturing activities since it measures quality. Inspection planning determines where, when, 
and how characteristics of a product are to be inspected. With the continued growth of product complexity and variety and the constant demand of reducing the product development cycle, industries are in search of more automated measurement operations, and integrated measurement knowledge. To assess quality, an actual, machined part is measured and geometric dimensioning and tolerancing (GD\&T) characteristics are assessed by comparing the designed (commonly referred to as "nominal") values and their actual values. Good parts maintain a fit and form within the specified GD\&T tolerance limits. Tolerancing is widely used in industry to define the allowable variation of discrete parts from their ideal shape.

The goal of this paper is to understand how quality information characterizing the manufactured parts can be reported in a Extensible Markup Language (XML) standardized format. The Quality Information Framework (QIF) is an American National Standards Institute (ANSI) standard sponsored by the Dimensional Metrology Standards Consortium (DMSC) that defines an integrated set of XML information models to enable the effective exchange of metrology data throughout the entire manufacturing quality measurement process - from product design to inspection planning to execution to analysis and reporting. The desire is that QIF will help foster a pervasive "digital thread" throughout the product lifecycle contributing to feedforward and feedback flow of quality information. The hope is that widespread adoption of QIF will lead to better and more optimized part design and manufacturing processes performance.

\section{Product Lifecycle Background}

The widespread use of computers and the pervasive networking of information have revolutionized manufacturing. The advantage of these technologies is that manufacturers now have the opportunity for end-to-end dissemination and accumulation of digital information. Yet, although the field of manufacturing is constantly improving and adopting innovative techniques, the transition to a completely digital enterprise is still only partially complete. The hope is that formal standards for digital representation throughout the enterprise will allow feedback and feedforward of manufacturing and product information throughout the enterprise. These digital standards must be well-understood, transcend the manufacturing domain into mainstream computer technology, yet at the same time have industry-wide focus and acceptance or the potential benefits are lost.

Product development is a complex process involving the integration of distributed resources, such as human beings, engineering tools, software systems, and a large variety of product-related machines. Figure 1 shows the basic sequential steps in the product lifecycle with no feedback consideration. Stark defines Product Lifecycle Management (PLM) as the business activity of managing, in the most effective way, a company's products all the way across their lifecycles; from the very first idea for a product all the way through until it's retired and disposed [2]. 


$\begin{gathered}\text { Customer } \\ \text { Requirements }\end{gathered}>\begin{aligned} & \text { Product } \\ & \text { Design }\end{aligned}>\begin{gathered}\text { Prototyping } \\ \text { Testing }\end{gathered}>\begin{aligned} & \text { Process } \\ & \text { Planning }\end{aligned}>$ Production $>$ Delivery $>$ Service $>\begin{aligned} & \text { Removal } \\ & \text { Disposal }\end{aligned}>>$

Figure 1 Product Lifecycle Steps

Of interest to this paper, is the product lifecycle that focuses on the "art to part" fabrication and assembly of a final sellable good. Figure 2 shows a high-level abstract view of the manufacturing activity product lifecycle. As such, we will focus on the product realization process, which is concerned with the design, fabrication, assembly, and inspection of the final product. More specifically the digital thread will be studied as both an upstream and downstream link to impart production information.

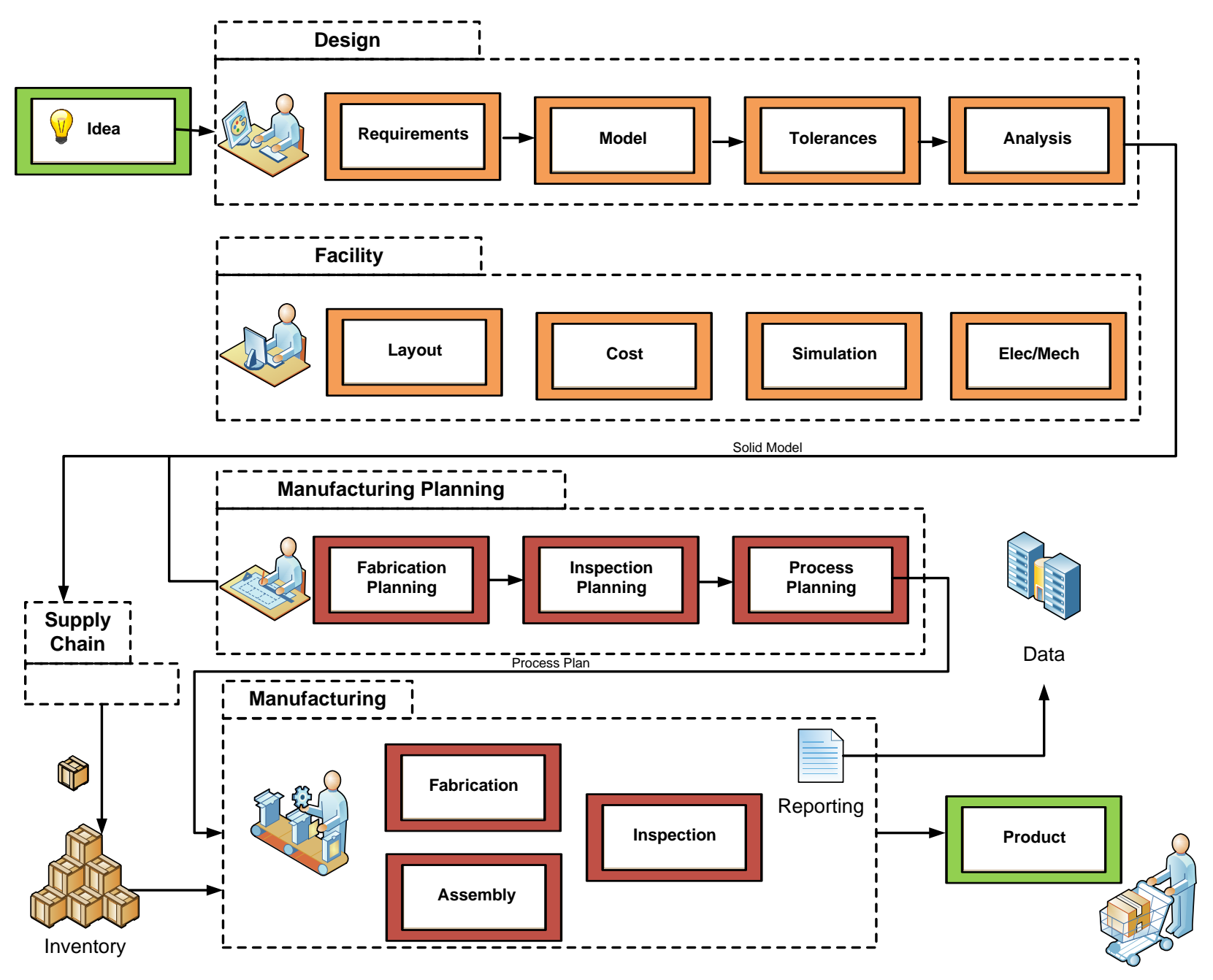

Figure 2 Manufacturing Activity in Product Lifecycle 
Product design spans an initial idea, model, refinement, and design, which requires cooperation of multidisciplinary design teams and the access to various engineering tools, such as computer aided design (CAD), finite element analysis (FEA), dynamic and kinematics analysis, simulation, and optimization packages, and databases.

Product manufacturing covers fabrication, machining, inspection and assembly. Production requires machines, transfer mechanisms, and supervisory control. Product engineering requires collaborative multidisciplinary engineering, including: computer integrated manufacturing (CIM), computer- aided manufacturing (CAM), computer-aided process planning (CAPP), computer-aided quality (CAQ), computer-aided inspection planning (CAIP), etc.

Product development has historically been a highly sequential activity where manufacturing was disconnected from engineering activities. Globalization and the rapid evolving of network tools, such as the Internet and web-based technologies, have revolutionized the product development process. Collaboration takes on a further importance if the manufacturing is geographically performed in different locations. Collaboration is possible throughout the entire product lifecycle by using lossless feedforward and feedback collaboration, but warrants a bi-directional digital thread.

The ability to collaborate by sharing product design and product data as well as manufacturing data among authorized users is now possible. With connectivity, the effective collaboration among designer, suppliers, and manufacturers throughout the entire product lifecycle is becoming much more important for continued competitiveness. The environment should not only automate individual tasks in the manner of traditional computer-aided engineering tools, but also mediate between individual tasks to promote collaboration within the context of a product design project [3]. In collaborative manufacturing, product lifecycle management (PLM) is recognized as a strategic business model to support collaborative creation, management, dissemination, and use of product assets, including data, information, knowledge, etc., across the extended enterprise from concept to end of life-integrating people, processes, and technology [4].

To date, two-dimensional (2D) product information has seen its capabilities far exceeded by threedimensional modeling and analysis systems, yet 2D drawings still retain a dominant role as contracting documentation. However, the emphasis on product information has moved drawings from 2D hard copy support to three dimensional (3D) digital form. Product and manufacturing information (PMI) facilitates a thorough 3D annotation environment that allows the association of geometrical tolerance directly to the 3D model. Given this 3D digital thread world, manufacturing is primed to automate the connectivity from design to shop floor, especially the role of quality feedback in improving the design, fabrication, and assembly of parts. The benefits and issues of PMI and the product lifecycle with a standardized digital thread with PMI have been studied, but are not yet a prevalent practice [5] [6] [7] [8].

This document will give a background on product life cycle with an emphasis on the role of quality, introduce QIF as an XML standard representation and exchange format for part quality information, and then discuss the opportunities that QIF can play in a 3D digital thread world. The goal of this document is 
to use digital thread concepts in manufacturing for the incorporation of the QIF in the product lifecycle. Without doubt, quality is an important consideration, as QIF offers the following potential benefits:

1. Real-time manufacturing and especially quality data integrated into the design phase of the product lifecycle,

2. Lossless data feedforward from the design phase into the production quality phase.

\section{Metrology Issues}

As background, there are numerous terms used in metrology as applied to inspection measurement systems and measurement results. Accuracy, precision, trueness, bias, error, variability, tolerance, traceability, reliability, and uncertainty are each integral to understanding metrology concepts. We will present definitions to define the metrology concepts [9] since the terminology is confusing. These terms (particularly accuracy and traceability) are very often confused (and abused) in practice due to difference in meaning to the seller, buyer, and user of metrology instruments [10].

Any given measurement result is usually taken as the estimate [11]. No measurement is exact. Trueness is the closeness of a measurement result to the actual (true) value. Error is the difference between a measured value of quantity and its true value. Variability is an inherent part of measurement systems and measurements - no two measurements can be expected to be the same. There are two types of measurement error: systematic error and random error. Measurement bias is the systematic error that is associated with the fact that a measured value contains a consistent error. A random error is caused by inherently unpredictable fluctuations with the measurement device and when a measurement is repeated it will generally provide a measured value that is different from the previous value.

In metrology, measurement uncertainty characterizes the dispersion of measured values to the true value. The accuracy of a measurement system is the degree of closeness of measurements of a quantity to the true value. Precision is the closeness of agreement among a set of measurement results. Precision can also be defined with reference to a measurement number of decimal places. Clearly, representing a measurement with a millionth decimal place versus a tenth decimal place provides a more exacting result, but there is no guarantee that extra decimal places add accuracy.

Ideally a measurement device is both accurate and precise, with measurements all close to and tightly clustered around the true value. The accuracy and precision of a measurement process is usually established by repeatedly measuring some traceable reference standard. Traceability to a widely accepted standard permits comparisons of measurements among users. Reliability refers to the consistency of accurate results over consecutive measurements over time [12].

\section{Measurement Uncertainty}

Decision rules for proving conformance or non-conformance with specifications are clearly defined by international standards. A component dimension must be accompanied by a tolerance [13] giving a lower specification limit (LSL) and an upper specification limit (USL), while a measurement result must be accompanied by an estimate of measurement uncertainty (U) [14]. 
Accuracy, properly defined as measurement uncertainty [14], is a key performance indicator for metrology. The accuracy of the required measurement resources are key determinant factors for the selection of inspection methods and technologies. There is a very wide spectrum of physical scale and accuracy requirements for which inspection resources need to be selected, ranging from measurement of small parts to large, complex products. Regardless of the scale, the dimensional measurement results need to be accompanied by the statement of uncertainty as defined by "Guide to the Expression of Uncertainty in Measurement" (GUM) [14].

Process inspection is dictated by the capability of the process and inspection systems. The level of inspection effort is dictated by the risk of Type I or Type II errors. Depending on the industry sector, such risk is driven by performance, safety, and fit. The result of any measurement contains errors which contribute to the overall value of measurement uncertainty as shown in Figure 3.

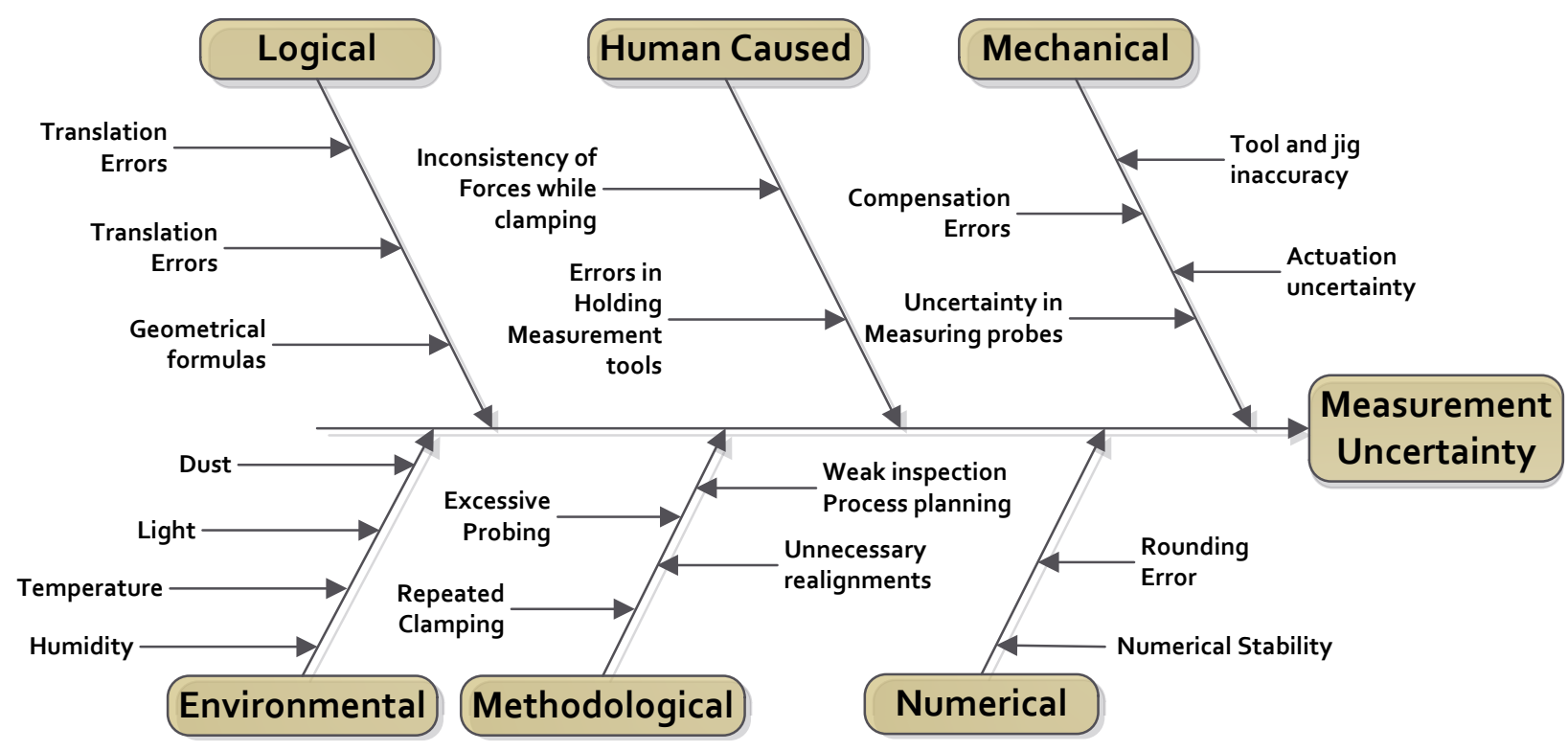

Figure 3 Ishikawa Cause Effect on Measurement Uncertainty

The standard ISO 14253 [15] makes it clear that the onus is on the supplier of the measurement data to guarantee the conformance to specification (tolerance) of the measurements, and that the data takes account of measurement uncertainty [16].

\section{Assigning Tolerances}

Variability, uncertainty, and tolerance have to be considered within product design to assure the required cost, performance, quality, and reliability. Tolerance is the allowable variance from the true dimension of a part. The importance of assigning product tolerances effects both cost and performance.

There is a well-known adage that states "Overtolerancing costs you money." On one hand, tight tolerances help assure satisfaction of the required specifications, but are more costly because the manufacturing process must be more precise, thus require more expensive manufacturing methods, more costly process, more steps, or more accurate fabrication machinery. On the other hand, loose tolerances may be cost- 
effective, but can lead to higher failure probability during manufacturing (e.g., scrap, rework) or breakdown during use.

Tighter tolerances not only contribute to cost, but add complexity which may make producibility more difficult. Milner et al. pointed out that "Customer requirements and overly conservative, and in some cases required, design margins tend to drive complexity into high-reliability systems which can adversely impact producibility by driving higher costs, longer manufacturing cycle times, and poor yields once the design is transitioned into production" [17].

Computer aided tolerancing can provide a simulation platform for modeling the effects of tolerance setting within a manufacturing process or assembly. Generally, tolerance analysis uses the worst-case maximum of dimensions and tolerances to calculate the maximum and minimum distance (clearance or interference) between two features or parts. Statistical tolerance analysis evaluates the maximum and minimum values based on some method for establishing the likelihood of obtaining the maximum and minimum values.

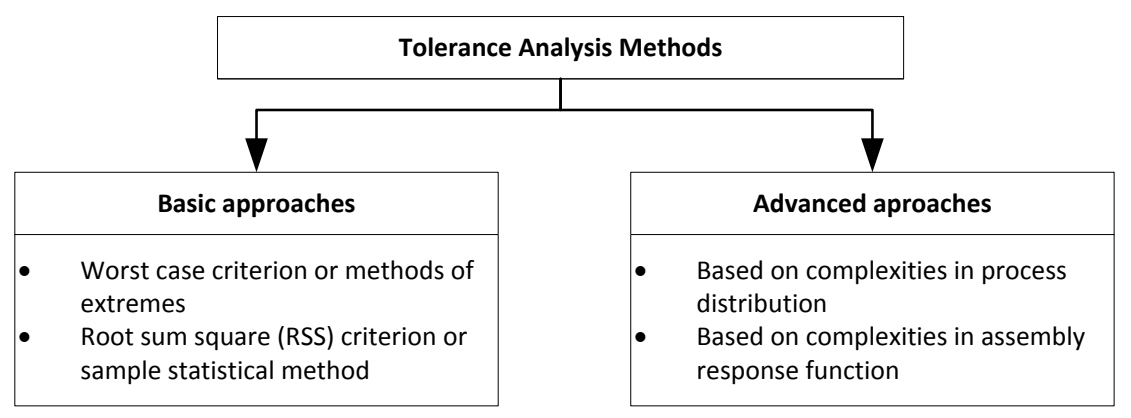

Figure 4 Tolerance Analysis ( [18] Used with Permission)

Tolerance-cost-optimization maximizes the product specifications tolerance while meeting an acceptable failure probability thus minimizing cost. Tolerance optimization during design has a positive impact on the production yields; with better yields reducing product cost and improving product quality. Tolerance-costoptimization uses either tolerance stackup analysis or a statistical tolerance analysis to compute the best tolerances.

When products fail to meet expectations, designers need to understand why the assigned tolerances failed to yield the exact part and assembly dimensions expected from manufacturing. Designers can then compensate for these errors in the next version of the product.

Discerning the reason for bad parts can be improved within a digital thread manufacturing environment through the use of quality feedback to help understand the manufacturing processes and product form and fit issues. It would be better yet, if advice were automated to show the designer explicit flaws and suggest potential improvements to the product.

Krogstie and Martinsen introduce Closed Loop Tolerance Engineering (CLTE) as a model that understands how actors, tools, and communication flow in collaborative design between the different main activities 
in product development [19]. The CLTE model has its origin in a survey within both the design and manufacturing units of a manufacturing company. The CLTE model has the following activities:

- Functional Requirements -The definition of the product to satisfy the customer, including the definition of acceptance limits for function and a rough selection of technical concepts.

- Tolerancing - All activities that lead to the definition of "limits of specifications" of product or process related parameters.

- Process Capabilities - The manufacturing processes to produce the product as specified.

- Product Performance - The validation of the functional behavior of the product through physical testing or simulation.

Given this model feedforward and feedback relationships are developed to associate the various elements in product development to tolerance engineering. Figure 5 shows the feedforward and feedback relationships within the CLTE model.

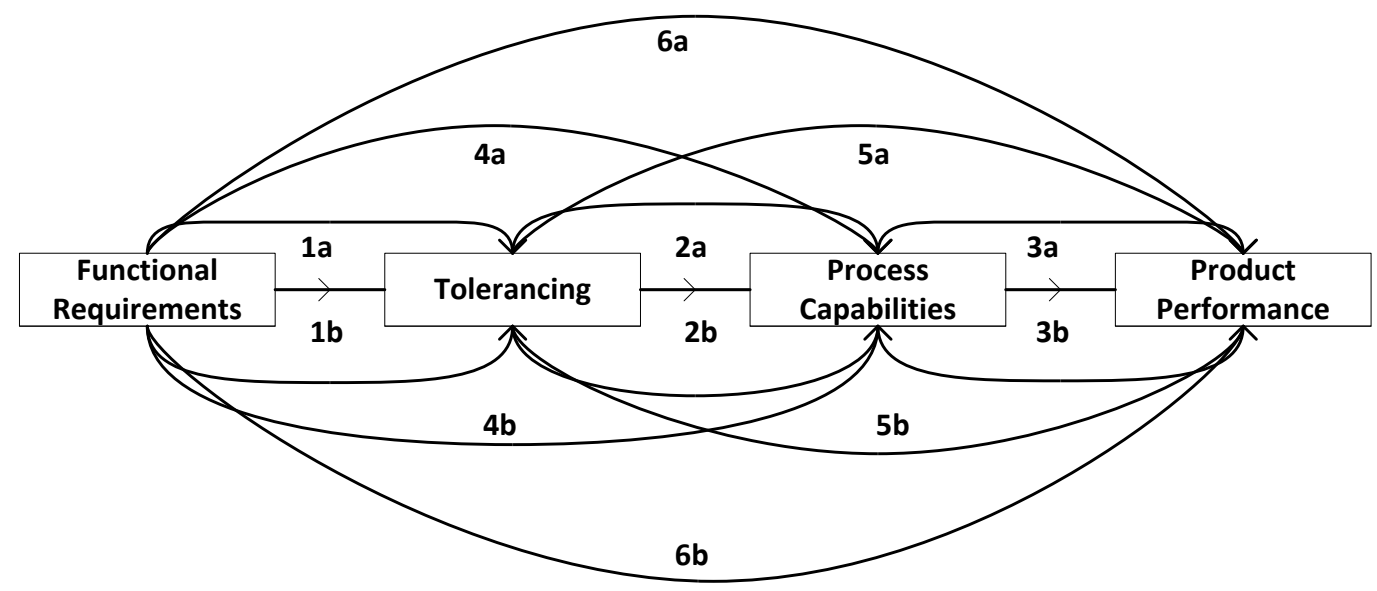

Figure 5 Activities and relations within CLTE [19] (Used with Permission) .

Merging the tolerancing language (GD\&T) with variation insight can lead to better tolerance analysis and more robust products. But tolerances, variation, and robustness are drawing from different sources of knowledge and require collaboration to share pertinent tolerance information. Figure 5 shows the relationships that form the basis of information distribution between activities. In the CLTE relationship diagram, actors can be designers, project leaders, management, manufacturing operators, test engineers, process engineers, foremen, etc. Tools and practices support the actors in making appropriate decisions when working with the four main activities. The lack of tools or wrong application of them can, in the same way, lead to inappropriate decisions. Table 1 presents the activities involved in the differing CLTE relationships. Of importance to the digital thread, is the automation of quality information flow in a CLTE model. As a first automation attempt at CLTE, the feedback relations pointing to the left in the CLTE model could be automated using QIF XML to allow reuse of existing tolerance knowledge. 


\begin{tabular}{|ll|}
\hline Relation & Activities \\
\hline $\mathbf{1 a}$ & $\begin{array}{l}\text { How are functional requirements transformed into specifications? Which actors translate such } \\
\text { requirements? }\end{array}$ \\
\hline $\mathbf{2 a}$ & $\begin{array}{l}\text { Given existing limits of specifications: How are the assumptions for the tolerances stored for } \\
\text { following projects? } \\
\text { of the process? }\end{array}$ \\
\hline $\mathbf{2 b}$ & $\begin{array}{l}\text { For each single parameter in a product or process: Are the critical parameters identified? Does } \\
\text { capability data exist? }\end{array}$ \\
\hline $\mathbf{3 a}$ & $\begin{array}{l}\text { Are the critical parameters for product performance known? How are the capability data for these } \\
\text { satisfactory? }\end{array}$ \\
\hline $\mathbf{3 b}$ & $\begin{array}{l}\text { Are sources of variation in product performance understood? Is knowledge gained in test } \\
\text { departments looped back? }\end{array}$ \\
\hline $\mathbf{4 a}$ & $\begin{array}{l}\text { Do functional requirements lead to a pre-definition of the manufacturing process? Is the } \\
\text { parameter possible to manufacture? }\end{array}$ \\
\hline $\mathbf{4 b}$ & $\begin{array}{l}\text { Can critical and less critical parameters be distinguished? Does capability data exist for } \\
\text { critical parameters? }\end{array}$ \\
\hline $\mathbf{5 a}$ & $\begin{array}{l}\text { For a given product or process: Are the key parameters (and their variation) influencing the } \\
\text { product performance known? }\end{array}$ \\
\hline $\mathbf{5 b}$ & $\begin{array}{l}\text { For a given product or process: Can variation in product performance be traced back to } \\
\text { variation in key parameters? }\end{array}$ \\
\hline $\mathbf{6 a}$ & \begin{tabular}{l} 
Can the chosen functional requirements be met by corresponding product performance? \\
\hline $\mathbf{6 b}$
\end{tabular}$\quad \begin{array}{l}\text { To what extent is existing knowledge from testing, simulation, and manufacturing relevant } \\
\text { for following projects? }\end{array}$ \\
\hline
\end{tabular}

The relationship and questions posed by the CLTE model form a basis for understanding the role of quality in the feedback and feedforward of production knowledge represented by the manufacturing digital thread.

\section{Lossless Data Exchange and Preventing Digital Tolerance Stack Up}

In a complete digital thread, all transfer of information is done through the use of "lossless" digital representation. Generally, lossless data exchange refers to compression techniques (such as Joint Photographic Experts Group (JPEG), or Moving Picture Experts Group (MPEG)) but in this case refers to the exchange of information from one stage in the product lifecycle to another. It may be preferable from the perspective of the Standard for the Exchange of Product model data (STEP) to use the STEP standard all the time for content as well as any translation. In theory, using the same representation will not result in any loss of information (or injection of errors).

Translation from one design language into another imparts variability into the design due to heterogeneous mapping of one representation into another. If the language translations imparted zero variability, the information would be identical; however, this is not usually the case. Digital tolerance stackup refers to the loss of precision in translating from one representation language to another (CAD$>$ STEP->QIF) and the requirement to increase the tolerance of the part design to compensate for the loss of precision. 
For example, a translated part must maintain a given tolerance of $+/-0.05$, so it must decrease its tolerance zone to +/- 0.04 to accommodate the potential imprecision of 0.01 due to the translation from language $A$ to language $B$. Thus, an acceptable translation from CAD into STEP may allow a +/-0.01 allowance around a box, which when stacked up with the translation of STEP into QIF means another loss of $+/-0.01$. In order for the part to maintain a manufacturability of $+/-0.05$, it has to decrease the tolerance of the original part to $+/-0.04$ and then to $+/-0.03$ to accommodate the loss of accuracy. Stacking two translations means a loss of +/- 0.01 for each translation and a loss of +/- 0.02 precision total, which corresponds to a decrease in tolerance of $+/-0.01$ for each translation. So the effect is a decrease in tolerance (which costs money) for each loss of precision for each translation.

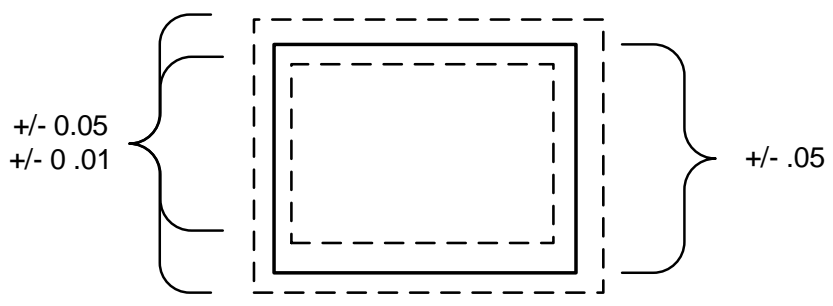

Figure 6 Digital Tolerance Stack Up

There has been much activity in developing manufacturing open standards: STEP [20], JTOpen [21], and QIF [22]. Despite all the effort in open standards, adoption has been slow. One of the major reasons for this is that meaning is often lost in data translation. Correct implementation of authoring systems and translators using product PMI associated with a CAD model such as geometric tolerances, dimensional tolerances, and datum features is essential for broader industry adoption of open standards. Lipman and Lubell discuss an approach used at NIST for checking the conformance of STEP files to the recommended practice for PMI representation [23]. Until CAD and PMI can guarantee a lossless translation between proprietary and neutral standard representations, manufacturers will be reluctant to attempt this action.

\section{Product Quality}

Quality needs to be initially defined in terms of characteristics, which vary from product to product. To ensure overall quality, delivered products must adhere to quality characteristics. Product quality is measured by its conformance to the design requirements including dimension, tolerance, and surface finish. The selection of a manufacturing process to produce a quality part depends on many constituent factors (machine tools, cutting tools, fixtures, process plan) and is greatly based on cost and process capabilities. Thus for example, the ability to produce a part depends on the machining of parts that adhere to given tolerances. Missing part tolerances are harmful as both cost and quality suffer. Clearly, no manufacturing process can make a perfect part, such that, designers must specify the acceptable variations to determine whether an actual part is a "good" part.

Today, symbols to represent GD\&T information on a part drawing have been standardized under different organizations, including ISO [24], as well as the ANSI Y14.5 [25] The standard GD\&T symbols provide a 
means for specifying the shape requirements of, and the interrelationships between, part features. GD\&T defines the nominal, as-modeled, or as-intended geometry. In GD\&T, a datum is a theoretically exact point, axis, or plane and serves as the origin from which the tolerance characteristics of features of a part are established. GD\&T specifications define the allowable datum variation by assigning tolerances, which are generally categorized into geometrical and dimensional tolerances. Geometrical tolerances are used to control the form, profile, orientation, location and runout of features. Dimensional tolerances are used to control the size and location of a feature referring to the datum. Form tolerances are applicable to single (individual) features or elements of a single feature; therefore, form tolerances are not related to datum. Form tolerance is a subset of geometric tolerances. It includes straightness, flatness, circularity, and cylindricity. This type of tolerance can be used for two distinctly different controls: surface control and control of derived median planes or derived median lines.

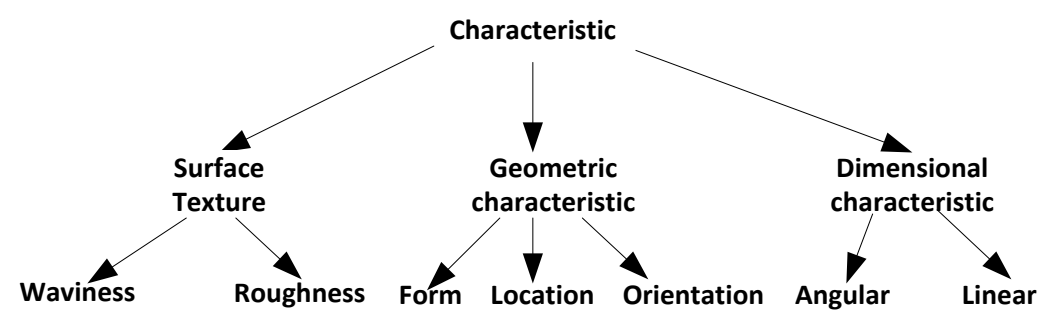

Figure 7 Dimension and Shape characteristics

Since drilling holes constitutes anywhere from $60 \%$ to $80 \%$ of machining operations, we will use a bolt pattern shown in Figure 8 as an example to explain the various interrelated modeling GD\&T elements. In three dimensions, holes are represented by cylindrical features typically contained within a block. Figure 9a shows a rectangular part with a hole at $(10,5)$ from the part origin (left hand bottom corner), and a 0.05 cylindricity $/ d$ specification defining the hole tolerance. The adjoining Figure $9 \mathrm{~b}$

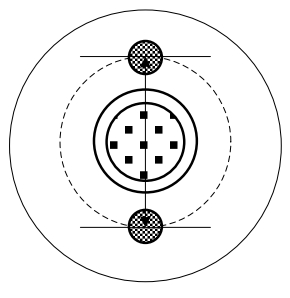
shows that this means that there is a tolerance zone for the cylindrical axis, and that three (3) circles generated from measuring points on the top/mid/bottom circles within the cylinder and the line generated from fitting these centers must be within $0.05 \mathrm{~mm}$ of the actual center line. 


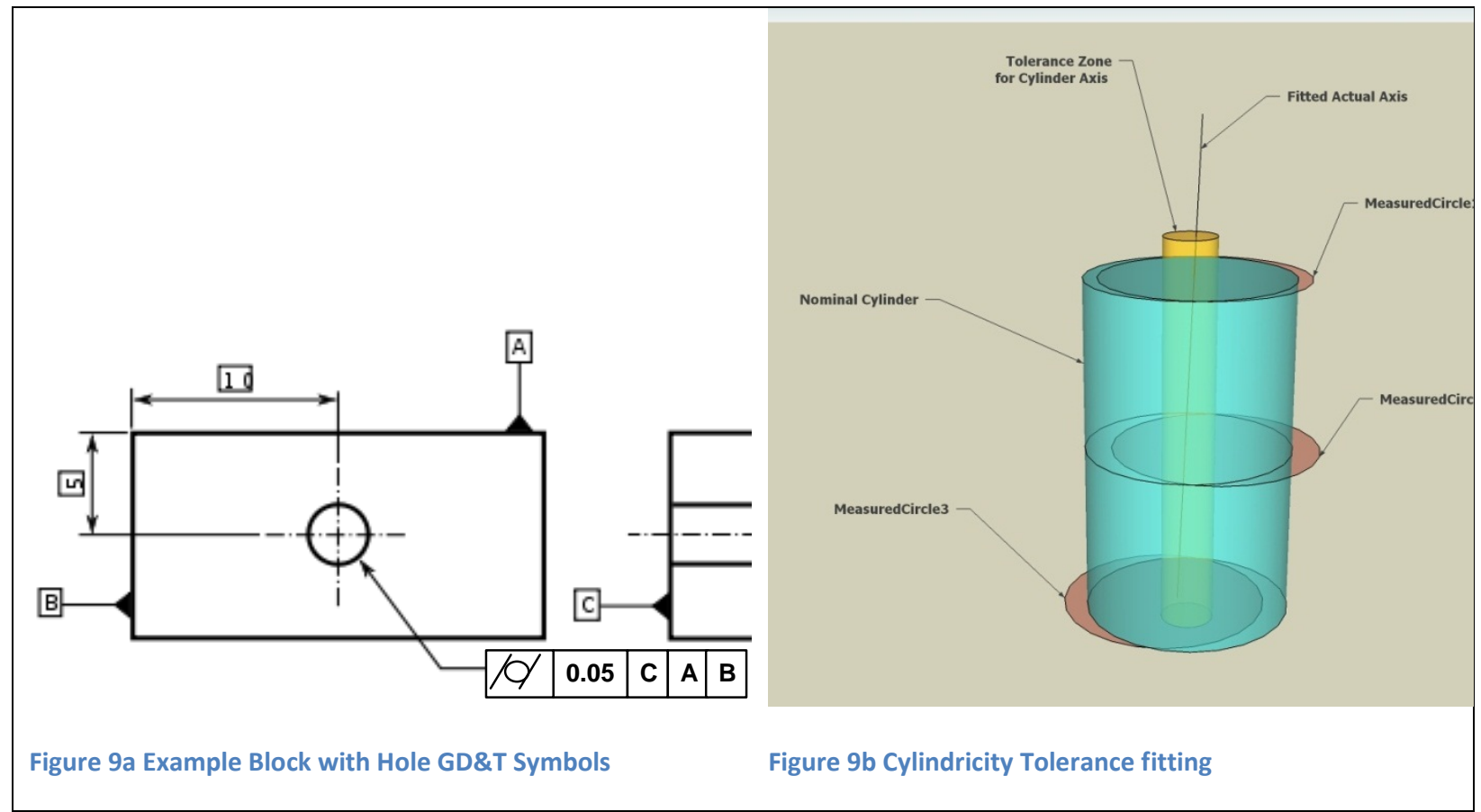

\section{Inspection Product Cycle}

Given this example, there are multiple phases of inspection. Figure 10 gives a brief overview of the main information modeling aspects of quality and the relationship to manufacturing processes (part design and tolerancing, inspection planning, inspection, analysis, and reporting.) 


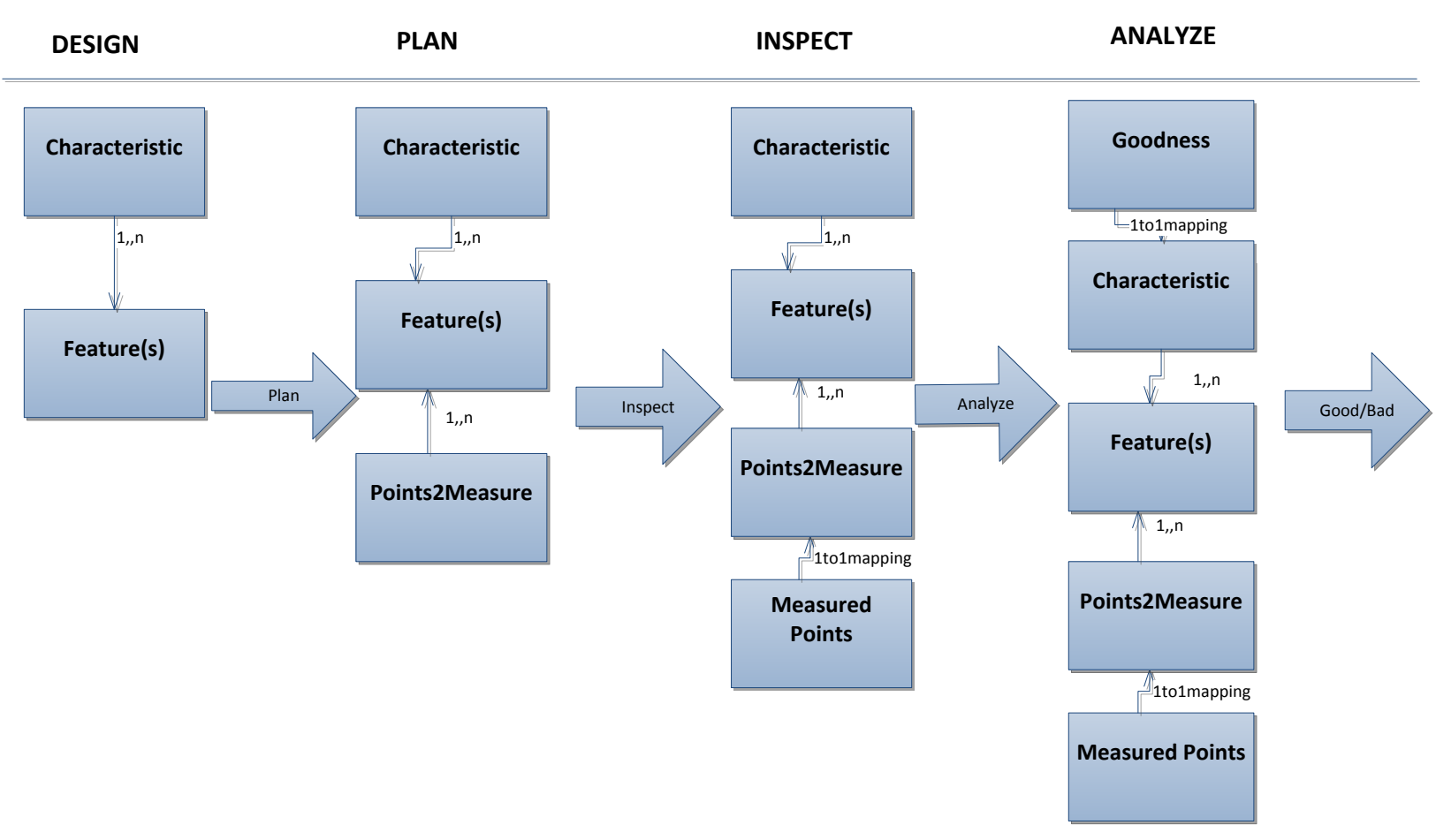

Figure 10 Manufacturing Phases of Inspection

DESIGN. In the Design phase, a characteristic (e.g., cylindricity tolerance) can be associated to one or more part features (i.e., the hole). In our terminology, a part means the part design, and a physical instance of a part is called an actual component.

PLAN. Once the feature and characteristic are developed, a Plan can be undertaken to assign nominal measurement points for the inspection device to probe. These nominal measurement points are assigned to the Feature Nominal, as defined in Figure 10 as the Point2Measure box.

INSPECT. Now an inspection can be done. Inspection using a measurement device produces data for the Measurement Points box which contains the probe readings corresponding to the nominal point lists. This Measurement Points could be generated by a simulation in the case that validation of tolerance planning and analysis is desired.

ANALYZE. An Inspection is performed and a set of points are recorded (MeasuredPoints in Figure 10). The recorded inspection points are contained in the Feature Actual measured data point set. Given the measured points, the part can be compared to the GD\&T specification to determine the goodness of the part.

Today, symbols to represent GD\&T tolerances on a part drawing have been standardized by different standards organizations. The standard GD\&T symbols provide a means for specifying the shape requirements of, and the interrelationships between, part features. Although automated inspection using GD\&T technology has become commonplace, the solutions for the planning, programming, and reporting 
of measuring data are still vendor specific, and as a result interoperability and quality results portability suffer.

\section{Quality Design}

Product design spans an initial idea, model, and refinement. This process requires cooperation of multidisciplinary design teams and access to various engineering tools (such as CAD, FEA, dynamic and kinematics analysis, simulation, and optimization packages), databases, and knowledge bases. Quality design specifies the part quality metrics by identifying features critical to fit and function first. The output is a list of features associated with tolerances and datum.

Product and manufacturing information (PMI) conveys information such as GD\&T, 3D annotation (text), surface finish, and material specifications. CAD systems that support STEP AP 242 Edition 2 [26] now have the capability to generate, save, or transmit tolerance information in a standard format.

The level of inspection required for any given feature is dictated by the risk of non-conformance. Depending on the industry sector, design risk is driven by performance, safety, cost, and fit. Manufacturing and inspection risks are dictated by the capability of the fabrication, inspection, and assembly systems. The measurement range, work volume, and precision of inspection resources dictate the ability to handle the part tolerance defined by the PMI.

\section{Quality Planning}

Quality planning uses the features and the corresponding characteristic tolerances to assign nominal measurement points for the inspection device to probe (number and location of feature points to measure) based on type of feature and the specific characteristic tolerance. With the continued growth of product complexity and variety, there is a constant demand for more sophisticated planning.

Our research emphasizes the use of standards to facilitate interoperable quality information exchange. Quality planning systems import the product model data with PMI, then import Resources and Rules information and generate/export Plans (whats and hows). Figure 11 shows the interplay of the various quality components in inspection/quality planning. 


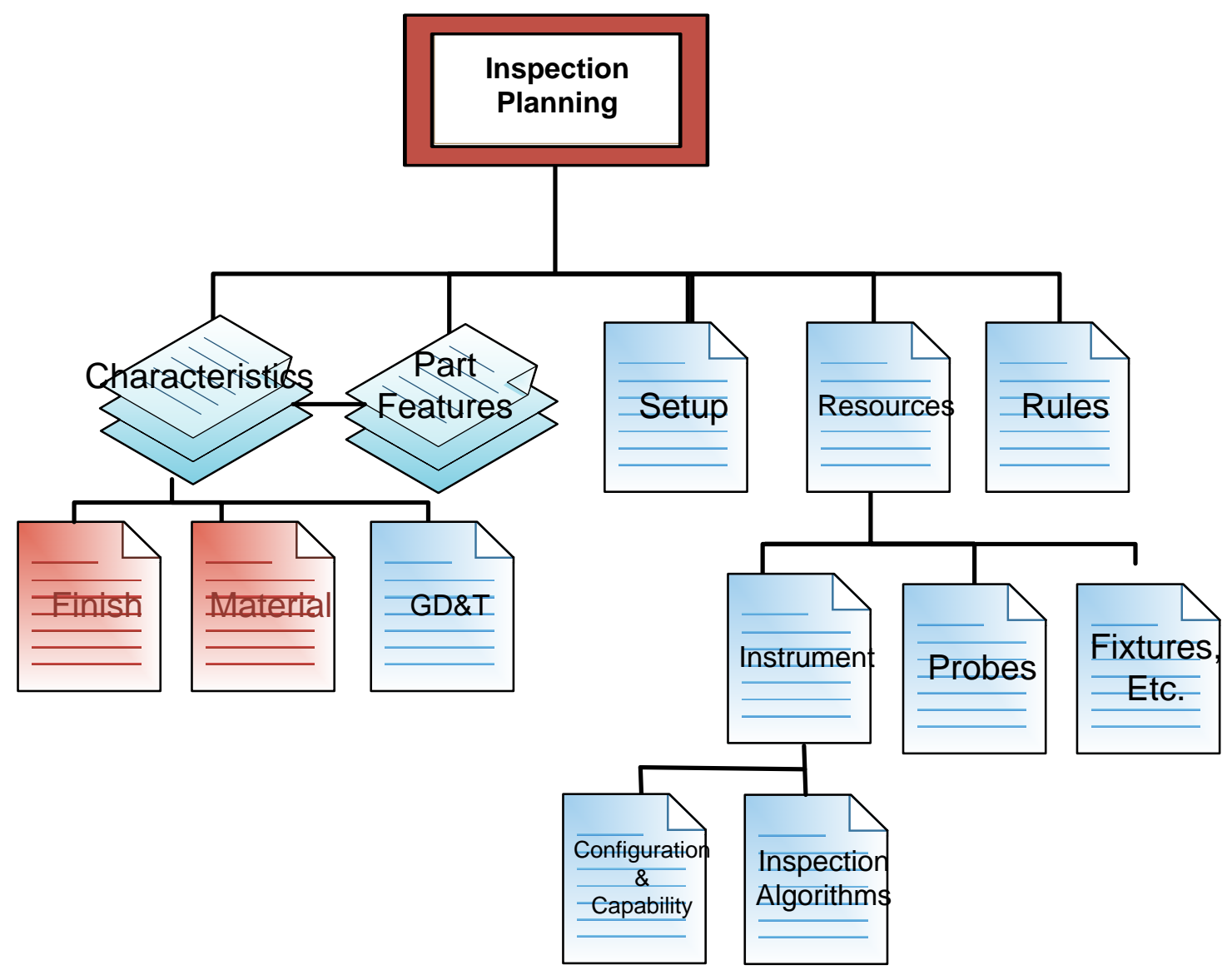

Figure 11 Inspection Planning

- Characteristic - a quality requirement such as dimension, tolerance, finish, material, or assembly, used in an inspection process. The importance of GD\&T has already been explained. The finish and material impact quality but are out of scope for this document. Figure 7 illustrates the hierarchical relationship of characteristics to texture, geometric, and dimensional tolerancing.

- Part Feature - defines a manufacturable feature.

- Resources - objects that perform or facilitate measurements. Clearly if a resource does not have the capability to measure to enough precision, then it is not a candidate resource.

o Probes - inspection of a given part requires a sensor to measure a particular feature. Different probes may be more suitable to different inspection features, and the matching of probe to feature/characteristic quality is part of the planning effort. Further, probe operation may be human hand-held or require a coordinate measuring machine. Also important when comparing probes, is measuring accuracy, precision, uncertainty, repeatability, and the gaging force specifications. These specifications should be matched to the requirements of the measuring and inspection operation.

o Instrument-may require a probe or be a gauge or coordinate measuring machine (CMM). Choosing the right CMM for any application requires considering several factors, including, the size, weight, and shape of the parts to be measured so that a CMM with 
the appropriate configuration, i.e., fixed or moving bridge, horizontal arm, cantilever, gantry, or portable is selected during planning.

- Rules - a specification of the type of measurement activity that should be carried out, given a condition context. For example, depending on the rules, an inspection device can measure 9, 15, or 1000 cylinder points to assess the quality. Rules are used to define the appropriate inspection procedures given the quality context.

- Setup - describes how the measurement components, such as part, measurement device, and inspection fixtures are to be physically organized.

\section{Quality Results}

Quality measurement performs part inspection, in which the actual measurement points are saved, with each actual point corresponding to the planned nominal point. Quality results represent parts as collections of production knowledge, which includes design, manufacturing, and inspection data. For example, a hole can be expressed with geometric design data for the hole location, diameter, and depth. A hole can also be associated with GD\&T data to ascribe the tolerance of the hole location, diameter, and depth as well as relationships to other features.

Quality results provides a mechanism to record measurement data generated from one or more inspections in a digital (computer-readable) format for all GD\&T elements. Elements include but are not limited to assembly information, part information, features, characteristics, nominal data, actual data along with traceability information that is collected during the inspection process such as operator identifier, and inspection environment attributes.

To assess the quality, the measurement data for a manufactured part and the corresponding GD\&T specifications are compared against the nominal values. Good parts maintain a fit and form within the specified GD\&T tolerance limits. Tolerancing is widely used in industry to define the allowable variation of discrete parts from their ideal shape.

Often the quality of the product must include traceability reporting to satisfy regulatory compliance. Traceability offers information about the circumstances of a quality measurement process, for example, the date, time, product instance, machinery, measurements, among others.

\section{Quality Analysis}

Manufacturing systems are by their nature imperfect. Inadequate control of process variability can lead to scrap, rework, and repair. Assuming a stable manufacturing environment, close control of production quality through frequent measurement and adjustment to process errors is desirable. Quality analysis compares the product measured dimensions to the nominal dimensions and based on the allowed tolerance will report the nature of the errors, sometimes over multiple parts, associated with each measured feature.

A major part of quality analysis is statistical process control (SPC), which as its name suggests is a statistical method of quality assessment. SPC uses techniques pioneered by Walter A. Shewhart at Bell Laboratories in the early 1920s [27]. SPC aims to detect quality (and by implication process) variation at any point of 
time. In the context of the quality life cycle, SPC uses statistical methods to analyze multiple sequential quality reports. SPC uses statistical methods to observe the quality of the performance of the manufacturing or design process (as measured by the variability) in order to detect significant variations before they result in sub-standard production.

Also as the name suggests, in SPC, sufficiently large numbers of samples have to be included in the analysis to render statistically meaningful results. The number of samples required could depend on several factors, such as the desirable/required confidence/reliability levels of the SPC results, the availability of the samples, and the time given for the SPC study.

These SPC techniques are based on measuring process output and using statistical methods to detect excessive variation. Results of the statistical methods are then summarized visually by control charts. SPC interpretation is based on assessing the variation in the studied process. It is expected that there will be some variation in a process. However, control chart rules should detect poor quality. It is then the onus of either or both the product design and manufacturing process to determine the source(s) and root cause of the variation; and remediate the cause.

SPC uses upper and lower control limits based on statistical standard deviation (i.e., $\sigma$ ) distances from the mean. A centerline bisects the upper and lower control limits. Some of the typical SPC rules, from the Western Electric set of rules [28], are typified by: 1 ) detection of any single data point falls outside the $3 \sigma$ limit from the centerline 2 ) two out of three consecutive points fall beyond the $2 \sigma$ limit, on the same side of the centerline; 3 ) four out of five consecutive points fall beyond the $1 \sigma$ limit, on the same side of the centerline; and 4) nine consecutive points fall on the same side of the centerline. SPC has other general performance metrics to detect erroneous error patterns (with or without associated control chart rule) including: 1) stratification - variation small relative to control limits; 2) mixture - tendency to avoid the centerline; 3) systematic - negative autocorrelation-a long series of observations that alternate highlow-high-low; 4) repetition - tendency of one chart to follow the same pattern as its predecessor; and 5) trends - sustained drift or shift in the long-term mean. Worst cases are that these statistical errors are large enough to render the manufacturing process incapable of producing the parts with required quality.

However, it is the association of bad quality to the source of the problem that can be difficult to resolve. Since most quality assessment uses established SPC algorithms, these techniques are for determining a problem, rather than resolving the problem. Further, much of the quality assessment and production feedback is manual. Instead, the use of advanced software technologies, such as machine learning or big data analysis, should be able to catalog and then analyze the results to produce feedback in an end-to-end quality chain. Further, the use of more modern technique should be researched to see if these technologies are amenable to not only detecting but associating problems to root causes.

Figure 12 shows the relationship among the various components in the analysis of quality information on the upstream and downstream product lifecycle. It is the rejection of parts based on quality and the automation of the constituent steps in dealing with the remediation that provides the clear cut benefits. The terminology used is as follows. C\&R refers to the cause and remedy. ECR refers to an engineering change request. PR refers to a problem report. There are some aspects of the quality information models 
that have been standardized, but complete standardization (and use of these electronic standards) to provide the necessary quality, manufacturing, and product information is not available at this time. This is the rationale for the mix of manual and automated modes.

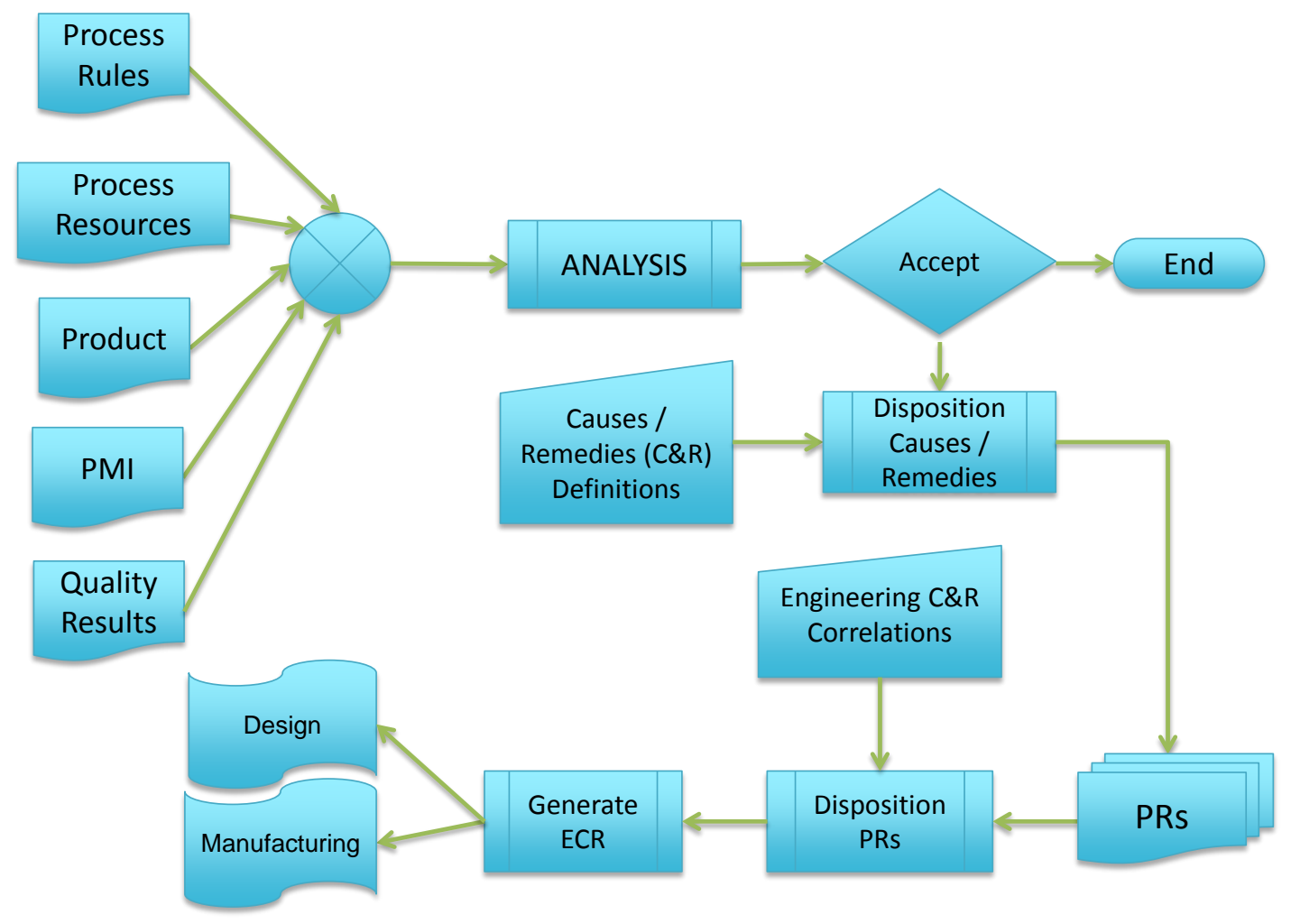

Figure 12 Quality Analysis and Feedback

Rules describe the best practices to be used in a production of the given product or assembly. Resources describe the hardware and software assets and processes used for manufacturing of a part. Results deal with recording of part measurements. Product and PMI include product definition and tolerancing information. Analysis deals with multiple part inspections and generally include statistics (such as those in SPC). Analysis is dependent on algorithms for comparisons and interpretation of the rules, resources, plans, and results. Acceptance of a statistical error depends on the underlying algorithms used to identify quality. For example, SPC control charts have some performance metrics for the evaluation of production. Analysis that yields non-compliance with SPC algorithms for trends, zones, and standard deviation result in rejection of parts. The rejection of parts leads to the cause and remediation steps. In Figure 12, Cause and Remediation Rules are assumed to exist, in order for the cause for quality non-compliance to be identified. If a matching $C \& R$ is found, then a problem report is issued detailing the production deficiency, the cause, and remediation. A C\&R is generic in nature and the $C \& R$ needs to be mapped into an ECR for either the engineering design or manufacturing units or both. 
An ECR is used to describe a suggested enhancement or problem with a product. Within the context of a quality lifecycle, an ECR originates from detected failure within quality analysis that can be remediated by an engineering change. Such failures include non-conforming part and assembly tolerances, repeatable part deficiencies, product enhancement ideas, and other various sources. The ECR could propose improvements with product components or assemblies, or the ECR could address manufacturing problems by specifying changes to the manufacturing process or equipment.

Currently, most of the remediation is achieved through the use of human knowledge, given the experience and understanding to interpret the quality problems. It is hoped that through the use of computer analysis, such as machine learning or big data techniques, the computer would understand and interpret quality problems and then initiate the ECR. This would imply that the computer can be taught the knowledge, patterns, and interpretations through viable machine learning techniques.

\section{Quality Information Framework (QIF)}

The Quality Information Framework (QIF) is an ANSI standard sponsored by the Dimensional Metrology Standards Consortium (DMSC) that defines an integrated set of Extensible Markup Language (XML) information models to enable the effective exchange of metrology data throughout the entire manufacturing quality measurement process - from product definition to inspection planning to execution to analysis and reporting. QIF handles feature-based dimensional metrology, quality measurement planning, first article inspection, and discrete quality measurement. QIF is gaining attention as an important quality technology [29] [30] [31] [32] [33] [34].

The Extensible Markup Language (XML) is a metalanguage, that is, a language used to create other $\mathrm{XML}$ languages. XML is typically used for defining a language to exchange data across the internet. With XML, the contextual meaning of the data can be represented to create a more application-specific language. The XML structure is a tree of elements containing branches of XML elements. An XML element is delimited by a start tag (ex: <ElementName $>$ ) and an end tag (</ElementName $>$ ) with CDATA being the nonmarkup character data (not XML tag related) between the tags. Elements can also have attributes, which are name-value pairs inside start tag (e.g.,: < PartDefinition id="partDefinition1">). The structure of XML is fundamentally tree oriented (a graph without any cycles). In QIF, the cross linking of information is done using unique identifiers (as shown by the "id" attribute).

QIF is based on XML, and uses terminology and semantics from the inspection world to represent the various elements in the QIF specification. The QIF information models are contained in files written in the XML Schema Definitions (XSD). The QIF XSD Version 2.0 models consists of six application schema files QIFRules, QIFResults, QIFPlans, QIFProduct, QIFStatistics, and QIFMeasurementResources bundled into a QIF Document. QIF also includes a library of XSD schema files containing information items used by all QIF applications (Auxiliary, Characteristics, Expressions, Features, GenericExpressions, Geometry, IntermediatesPMI, Primitives, PrimitivesPD, PrimitivesPMI, Statistics, Topology, Traceability, Units, and Visualization ). Figure 13 shows a high level perspective of the relationship among the different IF information models. At the core of the QIF architecture is the reusable QIF library which contains definitions and components that are referenced by the application areas. Around the QIF library core, 
Figure 13 shows the six QIF application area information models, Model-Based Design (MBD) which is equivalent to QIFProduct, Plans, Resources, Rules, Results, and Statistics. The "QIF Execution" model is, in the current version of QIF, a placeholder for future standardization and is now handled by the Dimensional Measuring Interface Standard (DMIS) standard [35]. The order of generation of QIF data generally proceeds clockwise around the diagram, beginning with QIF MBD and ending with QIF Statistics. Of note, users of the QIF information model are not required to implement the entire model.

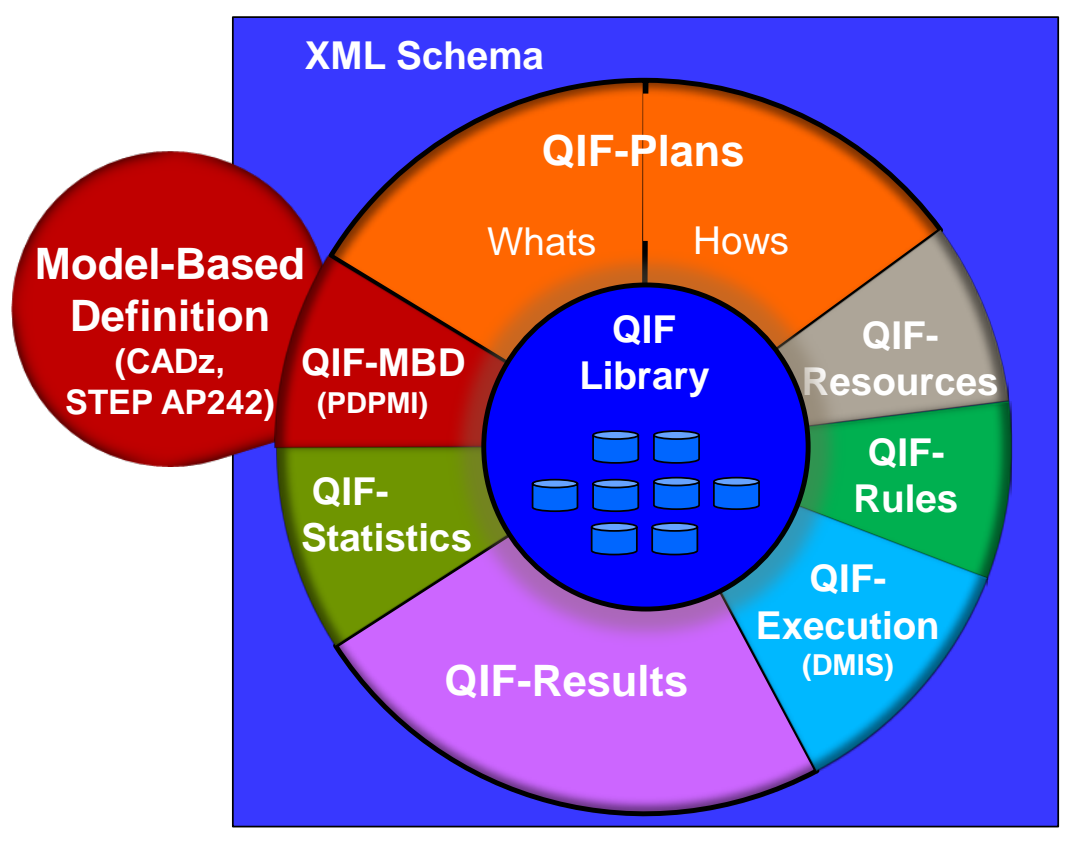

Figure 13 QIF 2 'Life Saver’ Architecture

The flow of QIF data starts with generation of CAD + PMI data exported as QIF Model Based Design (MBD) application data. Quality planning systems import the MBD and generate Plans (whats), then import Resources and Rules information and export Plans (whats and hows). Programming systems import Plans to generate Dimensional Measurement Equipment (DME) specific programs, or general instructions to guide inspection. Dimensional measurement equipment executes programs and evaluates characteristics of a single manufactured part or assembly and exports the measurements as Results. Analysis systems, typically performing statistical process control, import single parts Results and generate analysis of multiple part batches as QIF Statistics data.

QIF is intended to handle both lossless feedforward information translation, and cater to the ability to provide feedback integration to the product lifecycle in a unified and universal XML format. Currently, the translation from a CAD model based definition into the QIF format has been approached, and commercial products are available and standard processes are under development.

One goal of QIF is to satisfy the input specification requirements of GD\&T. Another goal of QIF is to satisfy the requirements derived from output results of quality assessment standards, such as AS9100, specifically AP9102A - First Article Inspection Report [36]. Users of the QIF information model are not 
required to implement the entire model. Any of the six application models may be used singly for exchange of quality data between software systems.

\section{QIF Software Development}

QIF is an extensive standard and fully defines most areas of quality information, including: quality measurement plans, measurement results, measurement rules, measurement resources, and results analysis. Currently, QIF has no competing standard or representation to represent measurement results, so QIF can be exploited in this area and offer many benefits. Reporting, recording, and analyzing QIF measurement results offer some promising opportunities.

AS9102 is the Society of Manufacturing Engineers (SME) Aerospace Quality Standard, known as, First Article Inspection Report (FAIR). The purpose of the First Article Inspection is to provide objective evidence that all engineering design and specification requirements are properly understood, accounted for, verified, and documented. The purpose of AS9102 standard is to provide a consistent documentation requirement for aerospace components First Article Inspection. QIF can be used to generate AS9102 reports and specific information for filling out a FAIR may be extracted from a QIF 2.0 XML instance file. The QIF standards process was especially committed to prove that QIF was sufficient in supplying FAIR information. The application of QIF to produce FAIR reports was demonstrated at the 2014 International Manufacturing Technology Show (IMTS) [37].

In exploratory research at the National Institute of Standards and Technology (NIST), QIF has been applied to a variety of applications. CodeSynthesis and the Xerces XML/XSD parser have been used to develop several QIF software programs useful in end-to-end quality management. These include parsing the QIF XML into native $\mathrm{C++}$ format, serializing the $\mathrm{C++}$ native format into QIF XML, generating Structured Query Language (SQL) [38] data tables from the QIF XSD, and storing QIF XML file instances into the SQL data bases. Figure 14 shows the block diagram of various components that were used in QIF software development. This QIF software development includes:

1. First, CodeSynthesis was downloaded, installed, and then used for $\mathrm{C}++$ code generation. This $\mathrm{C}++$ code was compiled and linked with Visual Studio 2010, and the application using the CodeSynthesis and Document Object Model (DOM) parsing of QIF was developed. Using the QIF explanation of XML First Article Inspection Reporting, Xerces XPath was used to generate FAIR report 1, and then more specialized code was developed to read and generate FAIR Report 3. The C++ application based on the CodeSynthesis output the FAIR report in HyperText Markup Language (HTML) that if opened by Google Chrome was able to produce a clean Portable Document Format (PDF) output.

2. The generated $\mathrm{C}++$ code was also tested by writing QIF results into the native $\mathrm{C}++$ representation, and then serializing the results into QIF XML.

3. Generation of SQL data definition language (DDL) was done to generate SQL data base tables. This software development was aimed at archiving the QIF XML. Although XML is a fine exchange language, saving the files into a data base is imperative for archival purposes. Given that XSD 
defines all the types, it seemed natural to map this specification into type and table SQL DDL and the read the QIF XML and insert this into the database using the XSD parsing knowledge.

SQL DDL is compatible with many open source data base providers, such as MySql and PostgreSQI. However, it was deemed of utmost importance that SQL tables support arrays within the fields. SQL has included this part of the standard since 2004, but some solutions (e.g., MySql) do not support arrays. The code was based on the fact that Xerces parsed the QIF XSD, however, the documentation describing the XSD parsing was quite limited and the representation from the parsing was also complicated. For this reason a symbol table was used to unwind the Xerces XSD parsing into a simpler format. Further, many advanced features not considered relevant to mapping QIF XSD to SQL DDL were ignored. QIF XSD exercised many features of XSD, but those XSD features that were not part of QIF were considered out of scope.

4. Generation of SQL insertion DDL was developed. The SQL DDL and QIF XML were done after Xerces parsed the QIF XSD and also parsed the QIF XML. At some point with a better understanding of the Xerces toolkit, the steps to unwind and decipher the Xerces mapping will be unnecessary.

5. The use of Open Database Connectivity (ODBC) was used to insert the QIF XML into the PostgreSQL database, instead of QIF DDL. ODBC is widely supported today, with drivers available for most platforms and most databases. Of note, the distinction between 64 and 32 bit ODBC caused some headaches. The main issue is insertion of QIF/XML requiring arrays or table entries. These insertions require an index from another preexisting table - so these insertions had to be completed before the "higher" level insertion was able to complete. 

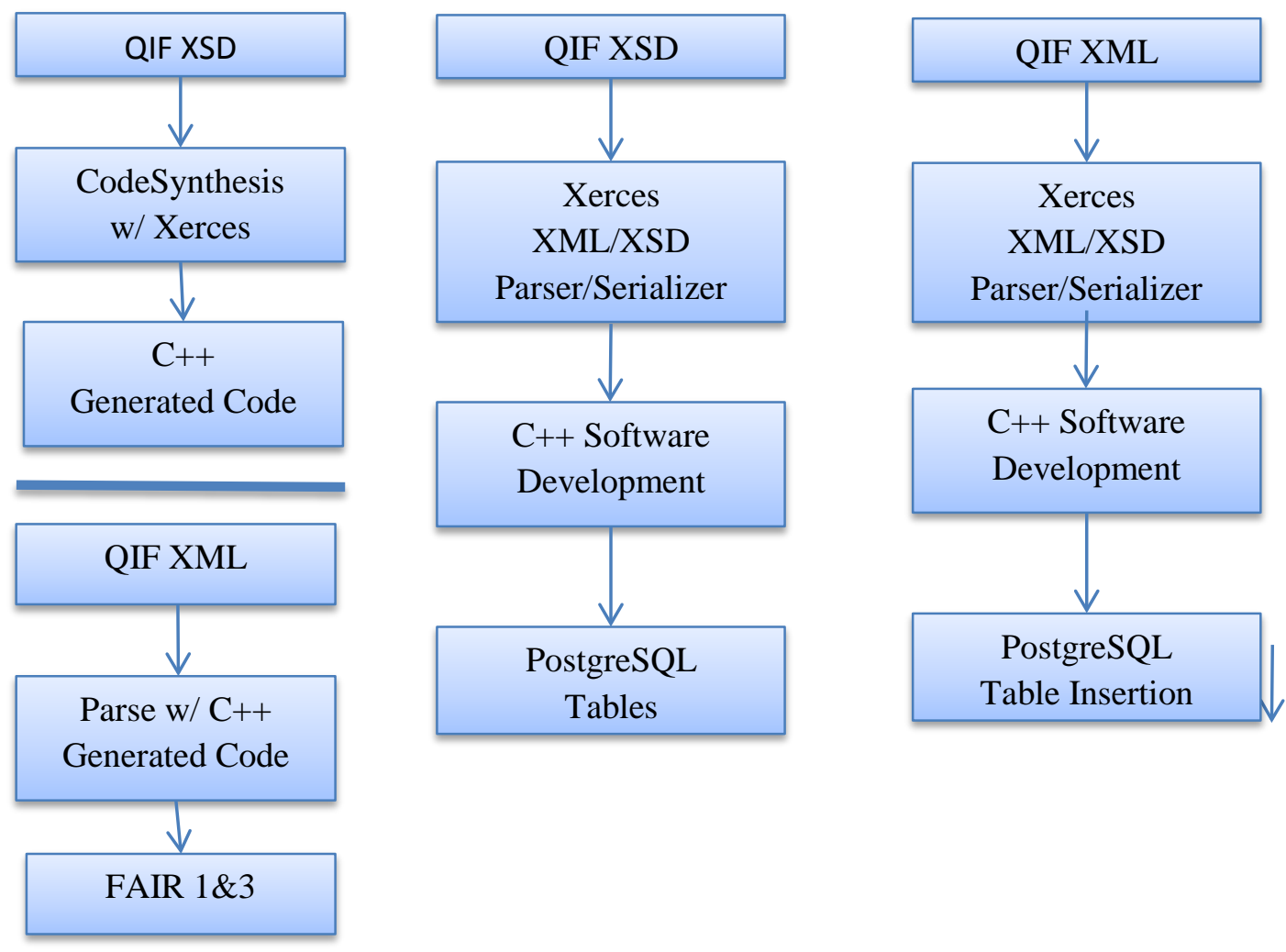

Figure 14 QIF XML Solution Space

The saving of multiple QIF XML files was considered important, so the saving of any QIF document into an SQL data base was developed. The open source PostgreSQL data base was selected since it supports arrays within SQL table fields. PostgreSQL allows columns of a table to be defined as variable-length multidimensional arrays. XML depends on the Element tag, which is a container object designed to store hierarchical data structures in memory. The element tag can have any number of child subelements and can be a list or array. Because of this common XML element software pattern, the mapping of a nested hierarchical list into an SQL array is quite straightforward.

In spite of reliance on SQL array structures, the number of SQL tables generated from the QIF schema was quite large (i.e., 1408). Further, although QIF XML was parsed and added to the SQL QIF data base, it is not clear how easily it is to access multiply derived substitution groups from the XML.

The existence of QIF alone does not guarantee success. Hopefully, helpful open source examples will assist manufacturers to understand the benefits of QIF and desire technology and solutions based on QIF.

There are many open source software projects that would help industrial partners understand and use QIF. These include:

1. Software to translate measurement output from one or more native commercial software quality systems into QIF 2.0 XML. Once proprietary quality measurements are translated into QIF XML, it 
is then straightforward to bundle and transmit the output into as an MTConnect "asset" [39]. Once in this QIFXML format, the data could be stored into the SQL data base.

2. The use of QIF XML to represent part features and quality inspections could be used to record defective part GD\&T measurements that correlate to a known (or several known) causes and then used to train a machine learning program to identify the cause of inspection deviations.

3. Regenerate QIF XML files from the SQL data base entries.

\section{QIF Enterprise Integration}

Recently, there has been interest in applying the web-enabled, real-time networking concepts to the communication of quality data in a standard XML format. Historically, the representation of quality data has been customized for the particular phase of manufacturing, including, design tolerances, measurement planning, inspection, recording/reporting, traceability logging, and statistical process control (SPC). The proliferation of data formats in the quality lifecycle is counterproductive, and Quality Information Framework (QIF) unifies the disparate quality related information models. With a common quality model, it is now cost-effective to disseminate quality information throughout the enterprise. Further, it is difficult to improve products and systems if they cannot be accurately measured and quantitatively characterized. In order to reduce costs, increase interoperability, and maximize enterprise integration, the MTConnect standards have been developed to "open" machine tools and factory floor devices for the manufacturing industry [40]. MTConnect is based upon prevalent web technology and provides free software development kits to minimize technical and economic barriers to MTConnect adoption.

Preliminary integration of the QIF quality standard and MTConnect has been done on a computer numeric control (CNC) system which shows the benefits of such information [39]. The lack of quality standards has led to time-consuming and inefficient production quality assessment with numerous opportunities for error and misinterpretation. Indeed, the use of MTConnect eases the integration of quality results into production processes with its use of XML and Internet communication web technologies. The role of MTConnect, inspection, and SPC was explored by Atluru and Deshpande [41]. A standalone SPC tool was developed that communicated with an on-machine probe and analyzed the inspection data to carry out a statistical analysis and then distribute the real-time results via an MTConnect network.

\section{Summary}

This paper reviewed the product life cycle and the role of quality in the design and manufacture of products. An emphasis was placed on the "digital thread" for pervasive networking of production information, especially quality. Of interest was the Quality Information Framework (QIF), which is an ANSI standard that defines an integrated set of XML information models to enable the effective exchange of metrology data throughout the entire manufacturing quality measurement process - from product definition to inspection planning to execution to analysis and reporting. The QIF library and QIF applications also provide generic templates for the user to instantiate for their own implementations, thus enhancing the code reuse and consistency for the implementations. The ongoing efforts of integrating 
MTConnect provide a mechanism to further expand integrated manufacturing into the design and manufacturing processes.

The migration of product data management from 2D paper and drawing based to standardized digital 3D model based is the goal of the digital thread project at NIST [42]. It is hoped that the benefits of an alldigital pervasive format will be apparent to manufacturers, in spite of perceived detrimental consequences of making the transition.

\section{Disclaimer}

Commercial equipment and software, many of which are either registered or trademarked, are identified in order to adequately specify certain procedures. In no case does such identification imply recommendation or endorsement by the National Institute of Standards and Technology, nor does it imply that the materials or equipment identified are necessarily the best available for the purpose.

\section{References}

[1] United Nations Industrial Development Organization, "Working paper - product quality: A guide for small and medium-sized enterprises," 2006.

[2] J. Stark, Product Lifecycle Management Decision Engineering, Springer London, 2011.

[3] Q. Hao, W. Shen, Z. Zhang, S.-W. Park and J.-K. Lee, "Agent-based collaborative product design engineering: An industrial case study," Computers in Industry, vol. 57, no. 1, pp. 26-38, January 2006.

[4] X. Ming, J. Yan, X. Wang, S. Li, W. Lu, Q. Peng and Y. Ma, "Collaborative process planning and manufacturing in product lifecycle management," Computers in Industry, vol. 59, no. 2-3, pp. 154166, 2008.

[5] F. Ricci, J. Sauza Bedolla, M. G. and P. Chiabert, "PMI: a PLM Approach for the Management of Geometrical and Dimensional Controls in Modern Industries," COMPUTER-AIDED DESIGN AND APPLICATIONS, pp. 36-43, 2014.

[6] A. B. Feeney, S. P. Frechette and V. Srinivasan, "A Portrait of an ISO STEP Tolerancing Standard as an Enabler of Smart Manufacturing Systems," JOURNAL OF COMPUTING AND INFORMATION SCIENCE IN ENGINEERING , vol. 15, no. 2, 2015.

[7] F. Ahmed and S. Han, "Semantic Mismatches for Interoperability of Product and Manufacturing Information," International Journal of CAD/CAM, vol. 13, no. 2, 2013. 
[8] J. Camba, M. Contero, M. Johnson and P. Company, "Extended 3D annotations as a new mechanism to explicitly communicate geometric design intent and increase CAD model reusability," ComputerAided Design, vol. 57, pp. 61-73, 2014.

[9] R. A. Fletcher, G. W. Mulholland, M. R. Winchester, R. L. King and D. B. Klinedinst, "Calibration of a Condensation Particle Counter Using a NIST Traceable Method," Aerosol Science and Technology, vol. 43, no. 5, pp. 425-441, 2009.

[10] R. D. Larrabee and M. T. Postek, "Precision, accuracy, uncertainty and traceability and their application to submicrometer dimensional metrology," Solid-State Electronics, vol. 36, no. 5, pp. 673-684, May 1993.

[11] Joint Committee for Guides in Metrology (JCGM), "International vocabulary of metrology - Basic and general concepts and associated terms (VIM)," 2008.

[12] Wikipedia, "Metrology," [Online]. Available: https://en.wikipedia.org/wiki/Metrology. [Accessed 29 7 2015].

[13] BS EN ISO 1101, "Geometrical Product Specifications (GPS) - Tolerances of Form, Orientation, Location and Run-Out," 2005.

[14] ISO/IEC, "General Metrology. Part 3: Guide to the Expression of Uncertainty in Measurement (GUM)," 1995.

[15] International Organization for Standardization, "ISO 14253-1:2013 Geometrical product specifications (GPS) -- Inspection by measurement of workpieces and measuring equipment -- Part 1: Decision rules for proving conformity or nonconformity with specifications," 2013.

[16] P. Maropoulos and D. Ceglarek, "Design verification and validation in product lifecycle," CIRP Annals - Manufacturing Technology, vol. 59, no. 2, pp. 740-759, 2010.

[17] T. Milner, M. Volas and A. Sanders, "Systems Engineering Methodology for Linking Requirements to Design Complexity and Manufacturing Trade Space Constraints," Procedia Computer Science, vol. 16, pp. 947-956, 2013.

[18] P. Singh, P. Jain and S. Jain, "Important Issues in Tolerance Design of Mechanical Assemblies. Part 1: Tolerance Analysis," Proceedings of the IMechE Part B: Journal of Engineering Manufacture, vol. 223, no. 10, p. 1225-1247, 2009.

[19] L. Krogstie and K. Martinsen, "Closed Loop Tolerance Engineering - A Relational Model Connecting Activities of Product Development," in 45th CIRP Conference on Manufacturing Systems 2012, 10.1016/j.procir.2012.07.089, 2012. 
[20] ISO, "ISO 10303-1:1994 - Industrial automation systems and integration -- Product data representation and exchange -- Part 1: Overview and fundamental principles," International Organization for Standardization, 1994 .

[21] ISO, "ISO 14306:2012 Industrial automation systems and integration -- JT file format specification for 3D visualization," International Organization for Standardization, 2012.

[22] DMSC, "ANSI/QIF Part 1-2014: Quality Information Framework (QIF) - An Integrated Model for Manufacturing Quality Information Part 1: Overview and Fundamental Principles Version 2.0," Dimensional Metrology Standards Consortium, Inc. (DMSC), 2014.

[23] R. Lipman and J. Lubell, "Conformance checking of PMI representation in CAD model STEP data exchange files," Computer-Aided Design, vol. 2015, pp. 14-23.

[24] International Organization for Standardization, "ISO 1101:2012 Geometrical product specifications (GPS) -- Geometrical tolerancing -- Tolerances of form, orientation, location and run-out," 2012.

[25] American Society of Mechanical Engineers, "ASME Y14.5-2009 Dimensioning and Tolerancing Engineering Drawing and Related Documentation Practices," 2009.

[26] ISO, "ISO 10303-242:2014 - Industrial automation systems and integration -- Product data representation and exchange -- Part 242: Application protocol: Managed model-based 3D engineering," International Organization for Standardization, 2014.

[27] M. Wilcox, "Prediction and pragmatism in Shewhart's theory of statistical control," Management Decision, vol. 42, no. 1, pp. 152-165, 2004.

[28] A. H. a. M. Mathur, "A Very Simple Set of Process Control Rules," Quality Engineering, vol. 5, no. 2, pp. 21-29, 1991.

[29] B. Pippenger, "Three-dimensional model for manufacturing and inspection," in Proceedings ASME Turbo Expo 2013: Turbine Technical Conference and Exposition, San Antonio, 2013.

[30] Y. Zhao, T. Kramer, W. Rippey, J. Horst and F. Proctor, "An integrated data model for quality information exchange in manufacturing systems," in Proceedings of the 37th International MATADOR 2012 Conference, Manchester, United kingdom, 2013.

[31] Y. F. Zhao, J. A. Horst, T. R. Kramer, W. Rippey and R. J. Brown, "Quality Information Framework Integrating metrology processes," IFAC Proceedings Volumes (IFAC-PapersOnline), vol. 14, no. Part 1, pp. 1301 - 1308, 2012.

[32] T. Kramer, R. Stone, M. Hoffman, S. Hoffman and W. Rippey, "Design and Usage Guide for Version 0.92 of the Quality Information Framework Data Model and XML (Extensible Markup Language) 
Schemas," US Department of Commerce, National Institute of Standards and Technology Internal Report, 2012.

[33] B. Morey, "Metrology Takes Flight," MANUFACTURING ENGINEERING, vol. 152, no. 2, 2014.

[34] Y. Zhao, T. Kramer, R. Brown and X. Xu, Information modeling for interoperable dimensional metrology, Springer Science \& Business Media, 2011.

[35] ANSI, "ANSI/DMIS 105.2 Dimensional Measuring Interface Standard, Rev. 5.2, Part 1," American National Standards Institute, 2009.

[36] SME International, "AS9102 - Aerospace First Article Inspection Requirement Revision B," 6102014. [Online]. Available: http://standards.sae.org/as9102b/. [Accessed 228 2015].

[37] Dimensional Metrology Standards Consortium (DMSC), "IMTS 2014 - DMSC Demonstrates New QIF 2.0," 2014. [Online]. Available: https://youtu.be/TqeVfqYX3PA. [Accessed 137 2015].

[38] International Organization for Standardization, "ISO/IEC 9075 - 2:2011 Part 2: Foundation (SQL/Foundation). It contains the most central elements of the language and consists of both mandatory and optional features.," ISO, 2011.

[39] J. Michaloski, Y. F. Zhao, B. E. Lee and W. Rippey, "Web-enabled, real-time, quality assurance for machining production systems," in Proceedings of the 12th CIRP Conference on Computer Aided Tolerancing (CIRP CAT 2012), Huddersfield, UK, 2012.

[40] MTConnect Institute, "MTConnect Standard," [Online]. Available: http://mtconnect.org. [Accessed $2372015]$.

[41] S. Atluru and A. Deshpande, "Statistical Process Monitoring With MTConnect," in ASME 2012 International Manufacturing Science and Engineering Conference, 2012.

[42] National Institute of Standards and Technology, "Enabling the Digital Thread for Smart Manufacturing," [Online]. Available: http://www.nist.gov/el/msid/infotest/digital-threadmanufacturing.cfm. [Accessed 107 2015]. 